\title{
Spatial fluctuations of helical Dirac fermions on the surface of topological insulators
}

\author{
Haim Beidenkopf ${ }^{1}$, Pedram Roushan, Jungpil Seo', Lindsay Gorman', Ilya Drozdov, Yew San Hor², \\ R. J. Cava² and Ali Yazdani ${ }^{1 \star}$
}

\begin{abstract}
Surfaces of topological insulators host a new class of states ${ }^{1}$ with Dirac dispersion ${ }^{2-4}$ and helical spin texture ${ }^{5}$. Potential quantum computing and spintronic applications using these states require manipulation of their electronic properties at the Dirac energy of their band structure by inducing magnetism or superconductivity through doping and the proximity effect ${ }^{6-9}$. Yet, the response of these states near the Dirac energy in their band structure to various perturbations has remained unexplored. Here we use spectroscopic mapping with the scanning tunnelling microscope to study their response to magnetic and non-magnetic bulk dopants in $\mathrm{Bi}_{2} \mathrm{Te}_{3}$ and $\mathrm{Bi}_{2} \mathrm{Se}_{3}$. Far from the Dirac energy, helicity provides remarkable resilience to backscattering even in the presence of ferromagnetism. However, approaching the Dirac point, where the surface states' wavelength diverges, bulk doping results in pronounced nanoscale spatial fluctuations of energy, momentum and helicity. Our results and their connection with similar studies of Dirac electrons in graphene $\mathrm{e}^{10-13}$ demonstrate that although backscattering and localization are absent for Dirac topological surface states, reducing charge defects is required for both tuning the chemical potential to the Dirac energy and achieving high electrical mobility for these novel states.
\end{abstract}

Since the recent discovery of topological insulators, modifications of the bulk chemical compositions or deposition of surface adsorbates have been commonly used to tune their chemical potential into the bulk gap and close to the Dirac energy $y^{3,14-18}$. Such efforts are motivated not only by attempts to maximize the ratio of surface to bulk conductivity, but also by the theoretical proposals for realizing yet more exotic states, such as Majorana fermions, based on topological insulators ${ }^{6,8,9}$. Simultaneous proximity to magnetism or superconductivity as well as tuning the chemical potential to the Dirac point of the surface state band structure are required to test these important predictions. It is often assumed that any chemical doping that is used to manipulate these states would not disrupt transport of topological surface states because of their topological protection against backscattering. However, experiments on graphene, which is by now a well-studied electronic Dirac system, have demonstrated that nearby defects (adsorbates or those in the substrate) can result in random local gating of such two-dimensional systems. This behaviour results in a residual concentration of induced carriers in graphene, hence preventing the chemical potential from uniformly reaching the Dirac energy throughout the system, and scattering that also limits the mobility of Dirac electrons in that system ${ }^{19}$. Whether such phenomena are also relevant for topological Dirac surface states that are protected by helical spin texture (as opposed to sub-lattice pseudospin texture in graphene) is not yet known. Finally, it is not clear whether such perturbations, which are non-magnetic and electrostatic in nature, are more dominant than scattering from magnetic defects for the properties of spin helical topological surface states. Addressing these issues is therefore critical for both unravelling the fundamental properties of topological surface states and also finding effective methods of using these states for various applications.

Spectroscopic mapping with the scanning tunnelling microscope (STM) has proven a powerful technique to examine the spatial structure and scattering properties of topological surface states in several different topological insulators, from $\mathrm{Bi}_{1-x} \mathrm{Sb}_{x}$ (ref. 20) to those having a single Dirac cone in their surface band structure, such as $\mathrm{Bi}_{2} \mathrm{Se}_{3}$ and $\mathrm{Bi}_{2} \mathrm{Te}_{3}$ (refs 21,22). For non-magnetic scatterers, these experiments have shown evidence for the absence of backscattering between equal and opposite momentum states due to their helical spin texture. Unusual transmission of these states through crystalline barriers has also been reported ${ }^{23}$. Previous STM studies, however, have not probed the properties of topological surface states near the Dirac point of the surface band structure, in part because of the low density of states in the vicinity of that energy ${ }^{21,22}$. The absence of any signatures of scattering in STM measurements near the Dirac energy has been attributed to topological protection of these states against scattering ${ }^{21,22}$, although only direct backscattering is strictly prohibited. Whether these states show an unusual sensitivity to magnetic scattering ${ }^{24}$ (due to breaking of time-reversal symmetry) is also unsettled as there has not been any STM study contrasting such scatterers to non-magnetic defects.

Our experiments were performed using a home-built cryogenic STM that operates at $4 \mathrm{~K}$ in ultrahigh vacuum. Single crystals of $\mathrm{Bi}_{2} \mathrm{Te}_{3}$ and $\mathrm{Bi}_{2} \mathrm{Se}_{3}$ doped with either $\mathrm{Ca}$ or $\mathrm{Mn}$ were cleaved in situ before STM measurements were taken at low temperatures. Figure 1 shows STM topography and energy-resolved conductance $(\mathrm{d} I / \mathrm{d} V)$ maps for the different samples at various energies. All topographic images taken on the different compounds (Fig. 1a) show triangular depressions that are associated with the substitutional dopants in layers close to the sample surface ${ }^{25}$. These defects scatter the surface states and give rise to the energy-dependent spatial interference patterns seen in the conductance maps of Fig. $1 \mathrm{~b}$ and c. At low energies (Fig. 1d) the conductance maps are dominated by quenched spatial modulations of the local density of states (LDOS) on a length scale of a few tens of nanometres. Such features are ubiquitous to all the doped topological insulators studied here and are present regardless of whether the dopants are magnetic or not.

To determine the microscopic origin of the inhomogeneous LDOS of the topological surface states we show in Fig. $2 a$ and $b$ the STM spectra obtained over a range of energies at different locations on the doped $\mathrm{Bi}_{2} \mathrm{Te}_{3}$ and $\mathrm{Bi}_{2} \mathrm{Se}_{3}$ surfaces, respectively. From these

\footnotetext{
${ }^{1}$ Joseph Henry Laboratory, Department of Physics, Princeton University, Princeton, New Jersey 08544, USA, ${ }^{2}$ Department of Chemistry, Princeton University, Princeton, New Jersey 08544, USA. *e-mail: yazdani@princeton.edu.
} 

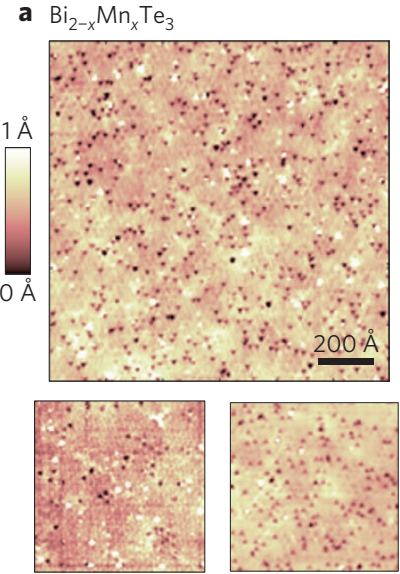

$\mathrm{Bi}_{2-x} \mathrm{Ca}_{x} \mathrm{Te}_{3}$

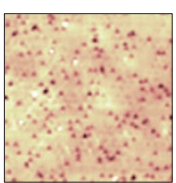

$\mathrm{Bi}_{2-x} \mathrm{Mn}_{x} \mathrm{Se}_{3}$ b
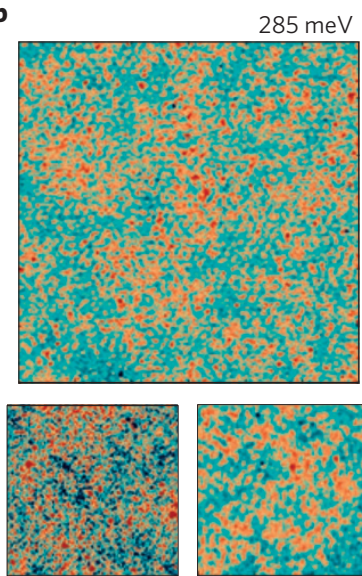

$340 \mathrm{meV}$

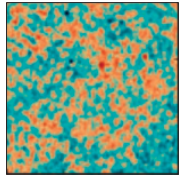

$285 \mathrm{meV}$
C
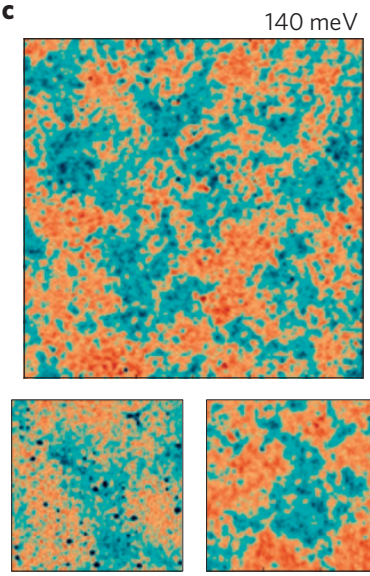

$140 \mathrm{meV}$

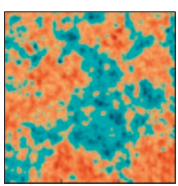

$150 \mathrm{meV}$ d
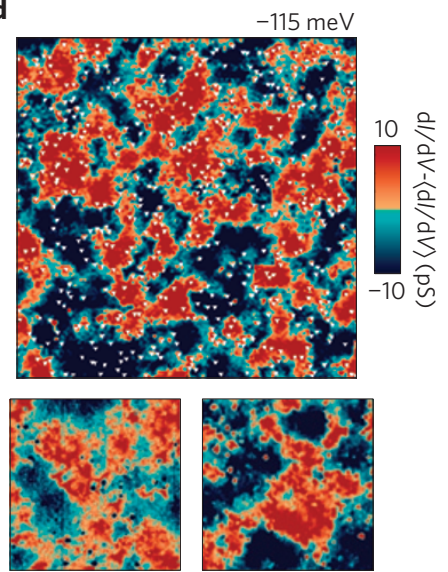

$-140 \mathrm{meV}$

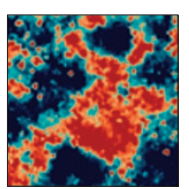

$-45 \mathrm{meV}$

Figure 1 | Interference and inhomogeneity on the surface of doped topological insulators. a, Topographic images on the surface of Mn- and Ca-doped $\mathrm{Bi}_{2} \mathrm{Te}_{3}$ and $\mathrm{Mn}$-doped $\mathrm{Bi}_{2} \mathrm{Se}_{3} \cdot \mathbf{b}-\mathbf{d}$, Conductance ( $\mathrm{d} / \mathrm{dV}$ ) maps taken at high, intermediate and low sample biases showing dispersive QPI patterns that ride a quenched inhomogeneity of the LDOS.
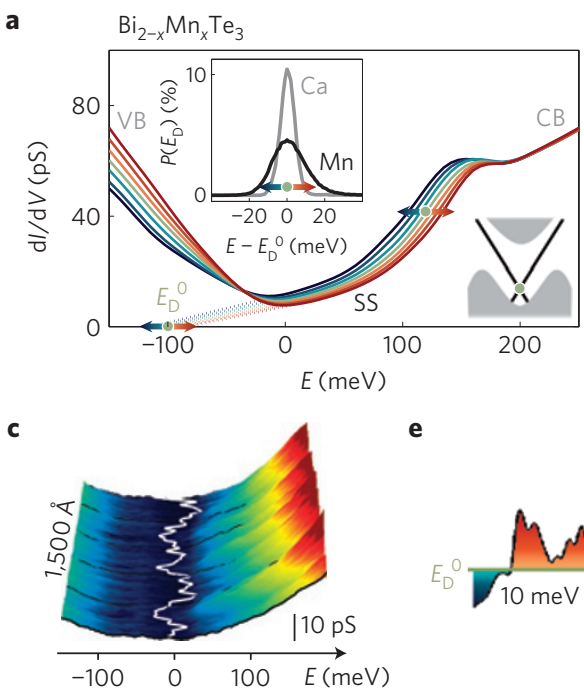

b

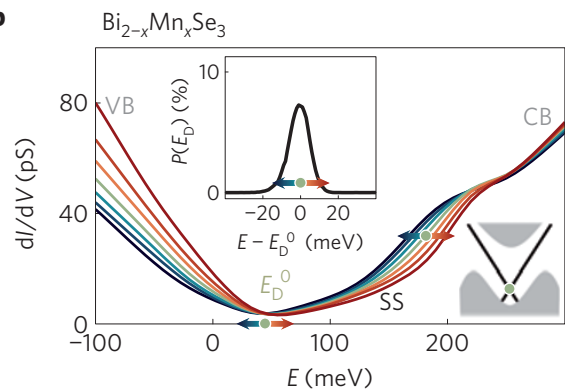

d
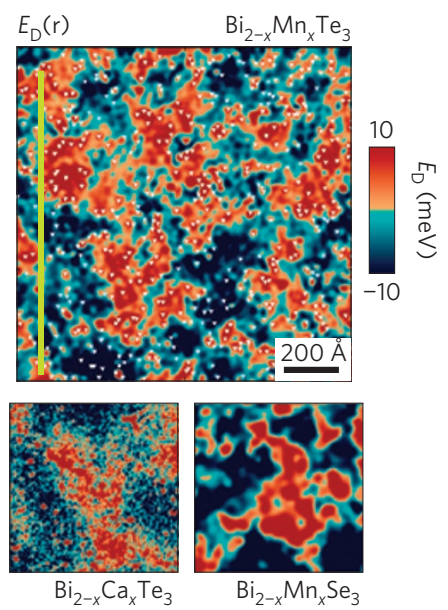

Figure 2 | Bulk origin of the charge inhomogeneity detected on the surface. $\mathbf{a}, \mathbf{b}$, The LDOS measured at various locations on the surface of Mn-doped $\mathrm{Bi}_{2} \mathrm{Te}_{3}$ and $\mathrm{Bi}_{2} \mathrm{Se}_{3}$, respectively, showing a rigid shift of the bulk and surface bands (band structures shown schematically). The Dirac point, $E_{\mathrm{D}}$ (approximated by linear extrapolation of the surface state's LDOS in $\mathrm{Bi}_{2} \mathrm{Te}_{3}$, and as the point of minimal LDOS in $\mathrm{Bi}_{2} \mathrm{Se}_{3}$ ), shifts with them. Insets show the Gaussian distribution of $E_{\mathrm{D}}$. c. High energy resolution LDOS, taken along a line cut that crosses several charge puddles (white line) on $\mathrm{Mn}^{-}$doped $\mathrm{Bi}_{2} \mathrm{Se}_{3}$, demonstrating the absence of resonances. $\mathbf{d}$, Spatial distribution of $E_{D}$ extracted from local shift in LDOS. Its structure shows no correlation with the locations of dopants (triangles). $\mathbf{e}$, Line cut along yellow line in $\mathbf{d}$ showing electron and hole 'puddles' that would form once the chemical potential is tuned to the average Dirac point, $E_{D^{0}}$, in the sample.

spectra it is evident that both the bulk and surface state bands are rigidly shifted in energy between different locations on the surface. We attribute these changes to bulk disorder, such as poorly screened charged deep dopants, as they are not necessarily correlated with the locations of the surface dopants resolved in STM topographs. The Dirac energy in the surface state electronic structure varies spatially together with the bulk-induced nanoscale fluctuations of the electronic band structure, as illustrated by the line in Fig. 2c. The extent of these variations can be mapped by extracting the energy shifts of the spectra obtained at different locations on the sample, as shown in Fig. 2d. Our measurements show that the Dirac energy follows a Gaussian distribution with a width of between 20 and $40 \mathrm{meV}$, depending on the specific dopant and its actual concentration. A cross-section of the bulk-induced energy landscape, shown in Fig. 2e, highlights that by tuning the sample's chemical potential close to the average Dirac energy, the local electronic structure alternates between electron-like and hole-like doped regions that would have opposing helical spin texture.

The 'puddled' real space electronic structures of the Dirac surface states are analogous to similar features observed in graphene by various scanning probe techniques ${ }^{10-12}$. Before describing a more quantitative analysis of these features and their comparison to similar behaviour in graphene, we show that these bulk-induced fluctuations have an important influence on the transmission of topological surface states. We determine the impact of bulk defects by examining how the underlying bulk-induced potential affects the response of these states to near-surface defects. Figure 3a-e shows the short wavelength quasi-particle interference (QPI) patterns for non-magnetically Ca-doped $\mathrm{Bi}_{2} \mathrm{Te}_{3}$. Remarkably, detailed Fourier analysis of the QPI patterns shows that short-wavelength scattering on the different samples is independent of whether the scattering dopant is magnetic or not (Fig. 3f-j and Supplementary Fig. S1) or 

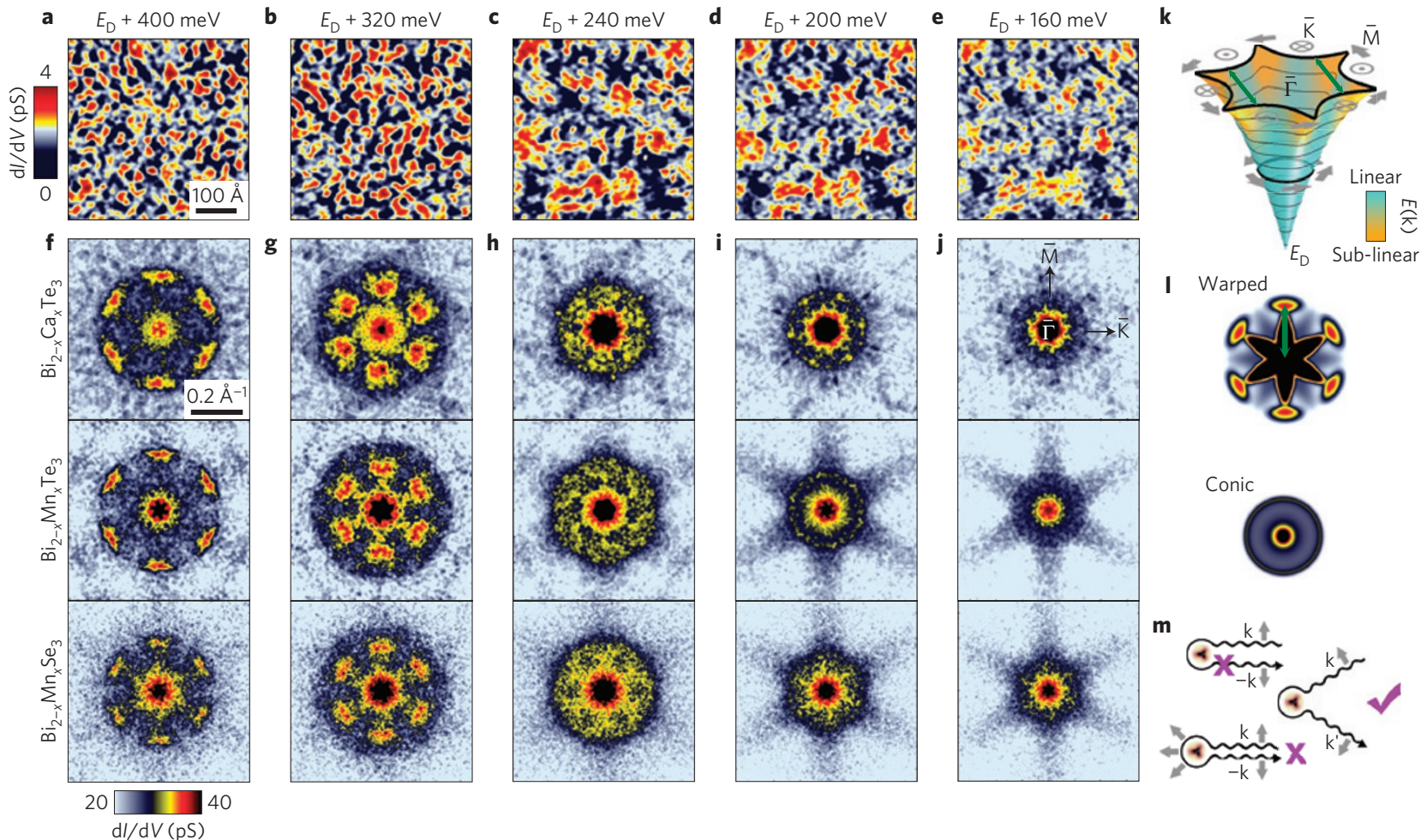

I Warped

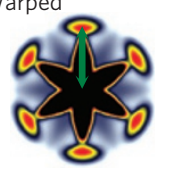

Conic

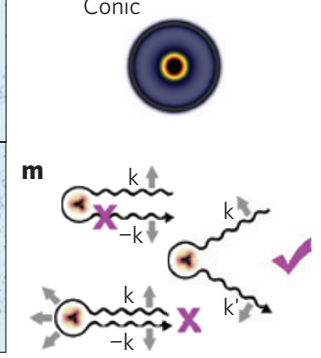

Figure 3 | Scattering of surface states. a-e, Real-space QPI patterns on the surface of Ca-doped $\mathrm{Bi}_{2} \mathrm{Te}_{3}$ at different energies. $\mathbf{f - j}$, Fourier transforms of the $\mathrm{QPI}$ patterns from $\mathrm{Ca}$ - and $\mathrm{Mn}$-doped $\mathrm{Bi}_{2} \mathrm{Te}_{3}$ and $\mathrm{Mn}$-doped $\mathrm{Bi}_{2} \mathrm{Se}_{3}$ given in top, middle and bottom panels, respectively. All compounds show similar patterns in q-space, consisting of six strong peaks along the $\bar{\Gamma}-\bar{M}$ directions at high energies and circular patterns at lower ones. $\mathbf{k}$, Schematic surface band structure in $\mathrm{Bi}_{2} \mathrm{Te}_{3}$ and the associated spin texture. Warping at high energies supports approximate-nesting conditions. Those along $\bar{\Gamma}-\bar{M}$ are indicated by green arrows. I, Calculated spin-dependent scattering probability (SSP) captures the Fourier space QPI patterns. $\mathbf{m}$, Illustration of processes and their contribution to the QPI pattern: helicity forbids backscattering off non-magnetic impurities (top) but allows oblique scattering and interference that have a finite overlap between initial and final spin states (middle). A magnetic impurity allows spin-flip backscattering but not interference of the initial and final spin states that remain orthogonal.

even if magnetic order is established ${ }^{25}$ (see Supplementary Fig. S2). In fact, the strong resemblance of the QPI patterns and their discrete Fourier transforms (DFT) across dopants (Ca and Mn) and materials $\left(\mathrm{Bi}_{2} \mathrm{Te}_{3}\right.$ and $\left.\mathrm{Bi}_{2} \mathrm{Se}_{3}\right)$ suggests that they can be understood on the basis of the shape of the Fermi surface and the spin texture associated with it ${ }^{2-5,26,27}$ (Fig. 3k). The warped shape of the surface band structure in $\mathrm{Bi}_{2} \mathrm{Te}_{3}$ and $\mathrm{Bi}_{2} \mathrm{Se}_{3}$ dominates scattering at high energies relative to the Dirac point. Fermi surface warping supports various approximate-nesting wave vectors, but it also induces an out-of-plane component to the spin texture that strongly attenuates the scattering peaks along the $\bar{\Gamma}-\overline{\mathrm{K}}$ direction ${ }^{27}$. Both of these can be accounted for in the calculated spin-dependent scattering probability (SSP; refs 20,23) presented in Fig. 31 (see Supplementary Fig. S3 for details). At low energies close to the Dirac point, in the region where the dispersion is conic, the scattering results in a simple circular pattern in the DFT of the real space QPI (Fig. 3i,j). These lowenergy scattering patterns are also consistent with the helical spin texture of the topological surface states that still allows all scattering processes other than direct backscattering ${ }^{28}$, illustrated in Fig. $3 \mathrm{~m}$ (See also Supplementary Information). Observation of these features requires improved signal-to-noise ratio when compared with QPI associated with the warped Fermi surface at higher energies.

To determine the influence of the bulk-induced disorder potential on the short-wavelength interference patterns caused by the surface impurities, we divide the real-space $\mathrm{d} I / \mathrm{d} V$ maps into sub-regions, as shown in Fig. $4 \mathrm{a}$, according to intervals of energy shift of the Dirac point in Fig. 2d. Obtaining the DFT from QPI measurements on two distinct regions (defined by the upper and lower halves of the distribution of Dirac energies) we find clear shifts of the q vectors of the QPI. An example of such a momentum shift $\left(\Delta q \sim 0.01 \AA^{-1}\right)$ is shown in Fig. 4b. Clearly, the surface Dirac electrons alter their wavelength to adjust to the underlying bulk disorder potential, and are not immune to such perturbations. Whereas such fluctuations in momentum are a relatively weak perturbation on the Dirac electrons at high energies, near the Dirac energy they are comparable to the average value of the momentum. Figure $4 \mathrm{c}$ indeed shows that the Dirac electrons exhibit such a shift in momentum, even close to the Dirac energy, where their dispersion is perfectly linear. Combining these results with the Fermi velocity obtained from the QPI dispersion $\left(v_{\mathrm{F}}=1.3 \mathrm{eV} \AA\right.$, in agreement with angular resolved photo emission spectroscopy (ARPES, ref. 4)) confirms that the extent of shift in momentum is consistent with the energy shift measured for the Dirac point $\left(\Delta E=v_{\mathrm{F}} \Delta \mathrm{q} \sim 16 \mathrm{meV}\right)$. Therefore, at energies close to the Dirac point in the presence of such fluctuations the sample is effectively made up of $\mathrm{p}-\mathrm{n}$ junctions ${ }^{29}$ and momentum carried by these electrons becomes ill-defined. The signature of this phenomenon can be seen in the absence of QPI patterns approaching the Dirac point when the wavelength of the topological surface states is comparable to or larger than the length scale imposed by the bulkinduced disorder. The lack of well-defined momentum near the Dirac point due to the fluctuations reported here is also likely to play a role in the apparent suppression of the ARPES-measured density of states in magnetically-doped topological insulators advocated recently ${ }^{15-17}$. Moreover, the similarity between fluctuations of near-Dirac electrons in both magnetically and non-magnetically doped samples signifies that the bulk-induced fluctuations (with $10 \mathrm{meV}$ variance) can have a far greater influence on the properties 


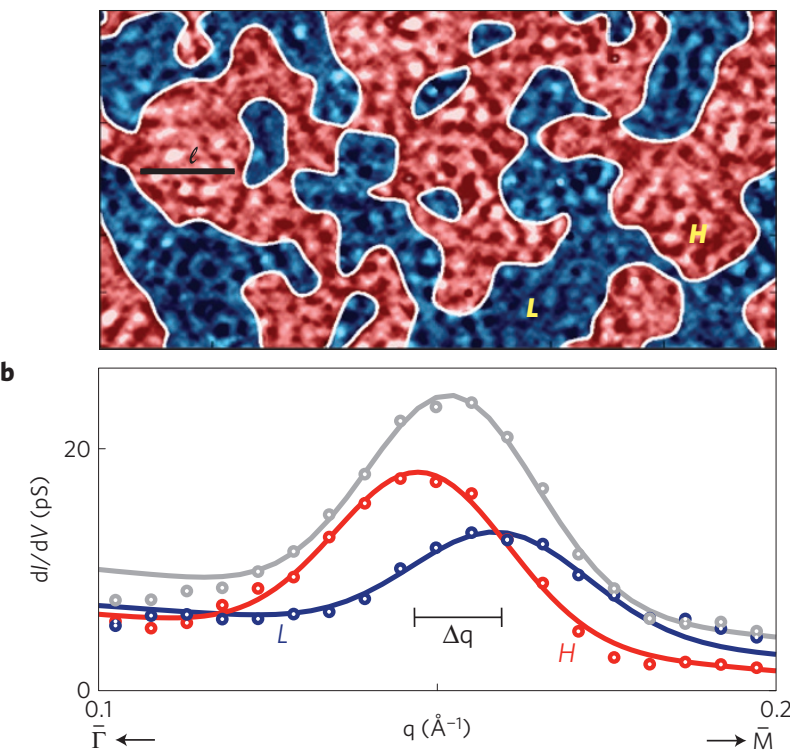

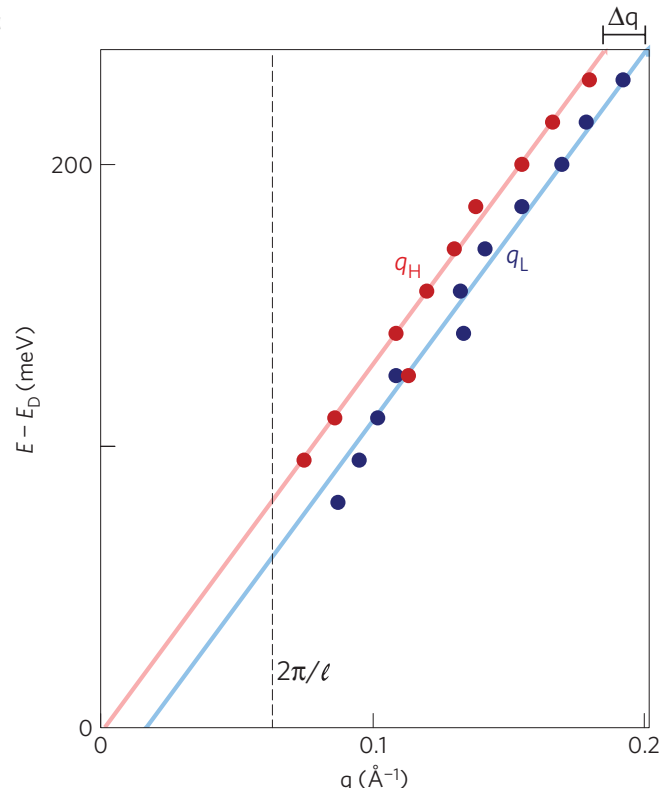

Figure 4 | Spatial fluctuations of momentum. a, The conductance map on $\mathrm{Bi}_{1.95} \mathrm{Mn}_{0.05} \mathrm{Te}_{3}$ divided into sub-regions with high and low LDOS. $\mathbf{b}$, Profile of a q-space QPI peak along $\bar{\Gamma}-\bar{M}$ (grey), and those peaks when Fourier transformed separately from the different sub-regions in a showing a relative shift in momentum $\Delta q=0.01 \AA^{-1}$ (lines are Gaussian fits to the data points). c, Similar analysis in the region of conical energy dispersion of $\mathrm{Bi}_{1.95} \mathrm{Mn}_{0.05} \mathrm{Se}_{3}$. QPI peaks from both sub-regions show linear dispersion shifted by an energy-independent $\Delta q \sim 0.01 \AA^{-1}$ with respect to each other. The dashed line marks $q=2 \pi / I$, below which the electronic wavelength is greater than the typical dimension of a puddle, I, causing the QPI pattern to vanish.

of near-Dirac electrons than the magnetic nature of scatterers (exchange energy is on the order of a few $\mathrm{meV}$ ).

The strong fluctuations of the momenta of the electronic states near the Dirac point, induced by the bulk disorder, raises the question of whether such disorder can localize topological surface states $^{28}$. The absence of resonances in the STM spectra (Fig. 2a-c), which one normally expects for electrons confined by a random potential on the nanoscale ${ }^{12,23}$, suggests that the Dirac electrons on topological insulator surfaces are not being localized by the bulkinduced disorder. Electronic states confined on the typical length scale of the bulk-induced potential, about $200 \AA$, would show a level spacing of about $70 \mathrm{meV}$-well within our STM spectroscopic resolution. The absence of any corresponding resonances in the data implies that the escape rate of the states from puddles is at least comparable to their level spacing, hence imposing a lower bound of $92 \%$ for the transmission of topological surface states through the random potential. These results put an upper bound on the possibility of backscattering, in a similar fashion to a previous study on topological surface states in Sb (ref. 22). Such an unusually high value may be indicative of Klein tunnelling - a phenomenon unique to Dirac particles in which they fully transmit through an arbitrarily large potential barrier ${ }^{30}$.

A more quantitative analysis of the influence of charge inhomogeneity caused by bulk disorder in topological surface states can be obtained by applying the theoretical model developed for a similar phenomenon in graphene ${ }^{13}$. In topological insulators, the bulk defects play the same role as those in the substrate supporting the graphene sheet, to cause fluctuations of the local band structure. Based on impurity density and crystal parameters the model estimates a Dirac energy variance of $16 \mathrm{meV}$ (see details in Supplementary Fig. S4), which agrees well with our experimental results based on analysis of STM data (Fig. 2a). The spatial correlation length of the puddles can be estimated by the Thomas-Fermi screening length in a Dirac system $L_{\mathrm{TF}}=2 \pi \kappa \hbar v_{\mathrm{F}} / e^{2} k_{\mathrm{F}} \approx 260 \AA$, where $\kappa$ is the effective dielectric constant and $k_{\mathrm{F}}$ the Fermi wavevector. It also agrees well with the length scale extracted from our spectroscopic maps of the Dirac point in Fig. $2 \mathrm{~d}$.
The physical picture emerging from our experiments and their analysis is that despite their protection against backscattering and localization, topological surface states are susceptible to charge disorder caused by bulk defects. This conclusion is consistent with recent transport studies on topological insulators, claiming that doped samples, besides having low mobilities, show magnetofingerprints associated with mesoscopic fluctuations on the length scale of a few tens of nanometres found in macroscopic single crystals $^{18}$. Transport experiments using electrostatic gating on thin films give evidence of a minimum conductivity that has been attributed to a defect induced residual charge density ${ }^{31}$. Both studies are consistent with our observation of bulk-induced fluctuations that introduce a length scale associated with screening of the defects and prevent tuning the chemical potential to the Dirac energy because of defect-induced charge puddles. Overall, similar to efforts in graphene, the reduction of bulk charge defects seems to be required to fully realize the potential of topological insulators in carrying out fundamental studies associated with the properties of this system near the Dirac point and for any potential applications that rely on high carrier mobility. Our quantitative analysis of puddle size and amplitude here suggest that the large bulk dielectric constant of topological insulators provides these systems with a natural advantage. In sufficiently clean samples we expect the fluctuation in the electronic structure to be ultimately much weaker when compared with those reported here.

Received 27 April 2011; accepted 7 September 2011; published online 9 October 2011

\section{References}

1. Moore, J. E. The birth of topological insulators. Nature 464, 194-198 (2010).

2. Zhang, $\mathrm{H}$. et al. Topological insulators in $\mathrm{Bi}_{2} \mathrm{Te}_{3}, \mathrm{Bi}_{2} \mathrm{Te}_{3}$ and $\mathrm{Sb}_{2} \mathrm{Te}_{3}$ with a single Dirac cone on the surface. Nature Phys. 5, 438-442 (2009).

3. Chen, Y. L. et al. Experimental realization of a three-dimensional topological insulator, $\mathrm{Bi}_{2} \mathrm{Te}_{3}$. Science 325, 178-181 (2009).

4. Xia, Y. et al. Observation of a large-gap topological-insulator class with a single Dirac cone on the surface. Nature Phys. 5, 398-402 (2009).

5. Hsieh, D. et al. Observation of unconventional quantum spin textures in topological insulators. Science 323, 919-922 (2009). 
6. Fu, L. \& Kane, C. L. Superconducting proximity effect and Majorana fermions at the surface of a topological insulator. Phys. Rev. Lett. 100, 096407 (2008).

7. Qi, X. L., Hughes, T. L. \& Zhang, S. C. Topological field theory of time-reversal invariant insulators. Phys. Rev. B 78, 195424 (2008).

8. Linder, J., Tanaka, Y., Yokoyama, T., Sudbø, A. \& Nagaosa, N. Unconventional superconductivity on a topological insulator. Phys. Rev. Lett. 104, 067001 (2009).

9. Rosenberg, G., Guo, H. M. \& Franz, M. Wormhole effect in a strong topological insulator. Phys. Rev. B 82, 041104 (2010).

10. Martin, J. et al. Observation of electron-hole puddles in graphene using a scanning single-electron transistor. Nature Phys. 4, 144-148 (2008).

11. Zhang, Y., Brar, V. W., Girit, C., Zettl, A. \& Crommie, M. F. Origin of spatial charge inhomogeneity in graphene. Nature Phys. 5, 722-726 (2009).

12. Jung, S. et al. Evolution of microscopic localization in graphene in a magnetic field from scattering resonances to quantum dots. Nature Phys. 7, 245-251 (2011).

13. Adam, S., Hwang, E. H., Galitski, V. M. \& Das Sarma, S. A self-consistent theory for graphene transport. Proc. Natl. Acad. Sci. USA 104, 18392-18397 (2007).

14. Hsieh, D. et al. A tunable topological insulator in the spin helical Dirac transport regime. Nature 460, 1101-1105 (2009).

15. Chen, Y. L. et al. Massive Dirac fermion on the surface of a magnetically doped topological insulator. Science 329, 659-662 (2010).

16. Wray, L. A. et al. A topological insulator surface under strong Coulomb, magnetic and disorder perturbations. Nature Phys. 7, 32-37 (2010).

17. Zhang, M. L. J. et al. Crossover between weak localization and weak antilocalization in magnetically doped topological insulator. Preprint at http://arxiv.org/abs/1103.3353 (2011).

18. Checkelsky, J. G. et al. Quantum interference in macroscopic crystals of nonmetallic $\mathrm{Bi}_{2} \mathrm{Se}_{3}$. Phys. Rev. Lett. 103, 246601 (2009).

19. Chen, J. H. et al. Charged impurity scattering in graphene. Nature Phys. 4, 377-381 (2007).

20. Roushan, P. et al. Topological surface states protected from backscattering by chiral spin texture. Nature 460, 1106-1109 (2009).

21. Zhang, T. et al. Experimental demonstration of the topological surface states protected by the time-reversal symmetry. Phys. Rev. Lett. 103, 266803 (2009).

22. Alpichshev, Z. et al. STM imaging of electronic waves on the surface of $\mathrm{Bi}_{2} \mathrm{Te}_{3}$ : Topologically protected surface states and hexagonal warping effects. Phys. Rev. Lett. 104, 016401 (2010).

23. Seo, J. et al. Transmission of topological surface states through surface barriers. Nature 466, 343-346 (2010).
24. Okada, Y. et al. Direct observation of broken time-reversal symmetry on the surface of a magnetically doped topological insulator. Phys. Rev. Lett. 106, 206805 (2011).

25. Hor, Y. S. et al. Development of ferromagnetism in the doped topological insulator $\mathrm{Bi}_{2-x} \mathrm{Mn}_{x} \mathrm{Te}_{3}$. Phys. Rev. B 81, 195203 (2010).

26. Lee, W. C., Wu, C., Arovas, D. P. \& Zhang, S. C. Quasiparticle interference on the surface of the topological insulator $\mathrm{Bi}_{2} \mathrm{Te}_{3}$. Phys. Rev. B 80, 245439 (2009).

27. $\mathrm{Fu}, \mathrm{L}$. Hexagonal warping effects in the surface states of topological insulator $\mathrm{Bi}_{2} \mathrm{Te}_{3}$. Phys. Rev. Lett. 103, 266801 (2009).

28. Biswas, R. R. \& Balatsky, A. V. Impurity-induced states on the surface of three-dimensional topological insulators. Phys. Rev. B 81, 233405 (2010).

29. Cheianov, V. V., Fal'ko, V. I., Altshuler, B. L. \& Aleiner, I. L. Random resistor network model of minimal conductivity in grapheme. Phys. Rev. Lett. 99, 176801 (2007).

30. Katsnelson, M. I., Novoselov, K. S. \& Geim, A. K. Chiral tunnelling and the Klein paradox in grapheme. Nature Phys. 2, 620-625 (2006).

31. Kim, D. et al. Electronic transport in the topological insulator regime: approaching the Dirac point in $\mathrm{Bi}_{2} \mathrm{Se}_{3}$. Preprint at http://arxiv.org/abs/1105.1410 (2011).

\section{Acknowledgements}

We gratefully acknowledge discussions with R. R. Biswas, S. Adam, and N. P. Ong. This work was supported by DARPA, ONR, NSF-DMR, and through NSF-MRSEC program through Princeton Centre for Complex Materials. The instrumentation and infrastructure at the Princeton Nanoscale Microscopy Laboratory are also supported by grants from DOE, the W.M. Keck foundation, and Eric and Linda Schmidt Transformative fund at Princeton.

\section{Author contributions}

Y.S.H. and R.J.C. carried out the growth of the single crystals and characterized them; STM measurements and data analysis were done by H.B., P.R., J.S., L.G., I.D. and A.Y. All authors discussed the results and contributed to the writing of the manuscript.

\section{Additional information}

The authors declare no competing financial interests. Supplementary information accompanies this paper on www.nature.com/naturephysics. Reprints and permissions information is available online at http://www.nature.com/reprints. Correspondence and requests for materials should be addressed to A.Y. 